\title{
表面增强拉曼光谱检测二噁英类化合物研究进展
}

\author{
程劼 王培龙* 苏晓鸥* \\ (中国农业科学院农业质量标准与检测技术研究所 北京 100081)
}

\begin{abstract}
摘要 以二噁英及二噁英类多氯联苯为代表的持久性有机污染物 $(\mathrm{POPs})$, 具有致畸、致癌、致突变的性质, 被国际癌 症研究中心列为人类一级致癌物. POPs 通过环境进入食物链对食品安全造成威胁和影响. 以表面增强拉曼光谱(SERS) 为代表的新型快速检测技术具有高灵敏分析的特点. 本综述总结了近年来基于 SERS 技术分析 POPs 的研究进展, 归纳 了不同类型增强基底，提出了 SERS 分析 POPs 的若干关键技术难点，并对未来 SERS 技术在 POPs 分析方面的发展进 行了展望.
\end{abstract}

关键词 表面增强拉曼光谱; 二噁英; 增强基底; 检测

\section{Recent Progress on the Detection of Dioxins Based on Surface-enhanced Raman Spectroscopy}

\author{
Cheng, Jie Wang, Peilong* Su, Xiaoou* \\ (Institute of Quality Standards and Testing Technologies for Agro-products, Chinese Academy of Agricultural Sciences, \\ Beijing 100081)
}

\begin{abstract}
Persistent Organic Pollutants (POPs), represented by dioxins and dioxin-like polychlorinated biphenyls have the property of teratogenic, carcinogenic and mutagenic, which have been classified as Group A human carcinogen by the international agency for research on cancer (IARC) and put into the initial list of Stockholm Convention managed by the United Nations Environment Program. POPs have posed a threat and impact on food security through the food chain from environment. The conventional detection methods, such as liquid chromatography-tandem mass spectrometry, high resolution gas chromatography-mass spectrometry and two-dimensional gas chromatography with time-of-flight mass spectrometry are sufficiently accurate, but fail to meet the requirements of on-site detection. Meanwhile, the rapid testing technologies for PCBs mainly included fluorescence detection, electrochemical sensors, and so on. As a new type of rapid detection technology, Surface-enhanced Raman Spectroscopy (SERS) has attracted significant attention as a promising analytical technique. With its ultra-sensitivity, high speed detection, ease of operation, SERS is particularly well-suited for the rapid detection of POPs. However, the multiple molecules in matrices may generate interfering Raman signals via competitive adsorption with the target compound on the substrate surface in the SERS detection of real samples. In addition, reproducibility represents a major bottleneck for the widespread application of SERS. Metal nanoparticle colloids are widely used as SERS substrates due to the hot spots formed between the nanoparticles. However, metal nanoparticle aggregation in colloidal solutions is difficult to control, leading to the random formation of hot spots. When the target POPs exist near the hot spots, the intensities of the enhanced Raman signals were unstable. Other factors influenced by the chemical adsorption such as vibration, charge transfer, and the deformation or distortion of molecules also affect the Raman signals. In the review, we provide an overview of the recent advances in SERS for POPs determination, especially the different types of enhanced substrates. And several key technical points of SERS detection including sensitivity, selectivity, and reproducibility have been summarized. Finally, the development of SERS for POPs detection in the future are proposed.
\end{abstract}

Keywords surface-enhanced Raman spectroscopy; dioxins; enhanced substrates; detection

\section{1 引言}

以二噁英及二噁英类多氯联苯为代表的持久性有 机污染物(POPs), 具有致畸、致癌、致突变的性质, 被 国际癌症研究中心列为人类一级致癌物, 具有 “世纪之 毒” 的称号, 极易在自然界扩散和动物体内蓄积, 被公
认为对人体健康具有极大潜在危害的全球性散布有机 污染物. 其中二噁英是 75 种多氯代二苯并二硻英 (PCDD)和 135 种二氯代二苯并呋喃(PCDF)的简称. 在 210 种二噁英类化合物中, 17 种 2,3,7,8-取代位同系物单 体具有较高的毒性当量(Toxicity Equivalency Factor, TEF), 通常是关注的重点. 在 209 种 PCBs 同系物中, 12

\footnotetext{
*E-mail: wplcon99@163.com; suxiaoou@caas.cn Received April 22, 2019; published June 5, 2019.

Project supported by the 13th five-year development plan of China by the National Key Research and Development Program (No. 2017YFC1600301) and the Fundamental Research Funds for Central Non-profit Scientific Institution, Chinese Academy of Agricultural Sciences (No. 1610072017006).

项目受国家十三五重点研发计划(No. 2017YFC1600301)和中国农业科学院基本科研业务费资助(No. 1610072017006).
} 
种具有类似二噁英毒性的共平面结构 PCBs 被称为二啞 英类 PCBs (dioxin-like PCBs, DL-PCBs)(表 1), 除此之 外, 在非二噁英类 PCBs 中, 六种指示性 PCBs (Indicator- $\mathrm{PCBs}$, In-PCBs) 被认为在环境介质和动物组织中暴 露风险较大 ${ }^{[1,2]}$. 国内外通常监测 In-PCBs 浓度来评估食 品中 PCBs 暴露水平.

表 129 种二噁英类化合物及 TEF 值

Table 1 TEF for 29 dioxin-like compounds (dioxin, furans and DL-PCBs)

\begin{tabular}{llll}
\hline \multicolumn{1}{c}{ 同系物 } & 毒性当量 & \multicolumn{1}{c}{ 同系物 } & 毒性当量 \\
\hline $\begin{array}{l}\text { 二噁英及呋喃 } \\
\text { (PCDD/Fs) }\end{array}$ & & $\begin{array}{l}\text { 二噁英类多氯联苯 } \\
\text { (DL-PCBs) }\end{array}$ \\
$2,3,7,8-\mathrm{TCDD}$ & 1 & & \\
$1,2,3,7,8-\mathrm{PeCDD}$ & 1 & Non-ortho PCBs & \\
$1,2,3,4,7,8-\mathrm{HxCDD}$ & 0.1 & PCB-77 & 0.0001 \\
$1,2,3,6,7,8-\mathrm{HxCDD}$ & 0.1 & PCB-81 & 0.0003 \\
$1,2,3,7,8,9-\mathrm{HxCDD}$ & 0.1 & PCB-126 & 0.1 \\
$1,2,3,4,6,7,8-\mathrm{HpCDD}$ & 0.01 & PCB-169 & 0.03 \\
OCDD & 0.0003 & & \\
& & Mono-ortho PCBs & \\
$2,3,7,8-\mathrm{TCDF}$ & 0.1 & PCB-105 & 0.00003 \\
$1,2,3,7,8-\mathrm{PeCDF}$ & 0.03 & PCB-114 & 0.00003 \\
$2,3,4,7,8-\mathrm{PeCDF}$ & 0.3 & PCB-118 & 0.00003 \\
$1,2,3,4,7,8-\mathrm{HxCDF}$ & 0.1 & PCB-123 & 0.00003 \\
$1,2,3,6,7,8-\mathrm{HxCDF}$ & 0.1 & PCB-156 & 0.00003 \\
$1,2,3,7,8,9-\mathrm{HxCDF}$ & 0.1 & PCB-157 & 0.00003 \\
$2,3,4,6,7,8-\mathrm{HxCDF}$ & 0.1 & PCB-167 & 0.00003 \\
$1,2,3,4,6,7,8-\mathrm{HpCDF}$ & 0.01 & PCB-189 & 0.00003 \\
$1,2,3,4,7,8,9-\mathrm{HpCDF}$ & 0.01 & & \\
OCDF & 0.0003 & & \\
\hline
\end{tabular}

1999～2011 年间，比利时、荷兰、法国、德国等国 相继发生的二噁英污染食品安全事件, 造成重大经济损 失和政治影响. 2016 2018 年期间，国内土鸡蛋，供港、 供台大闸蟹二噁英超标等食品安全事件, 使二啞英污染 食品安全问题备受关注; Nature Ecology and Evolution 近年来报道的在远离大陆, 距离平面 10500 米的深海生 物中发现 PCBs 含量近 $500 \mathrm{ng} / \mathrm{g}$ (按脂重计) ${ }^{[3]}$, 让世界震 惊. POPs 通过环境进入食物链(图 1), 对食品安全造成
威胁和影响, 对食品中二噁英污染的监控与安全监管迫 在眉睫.

目前针对 POPs 的仪器检测多采用气相色谱法 ${ }^{[4]}$, 气相色谱-质谱法 ${ }^{[5,6]}$, 全二维气相色谱-飞行时间质谱 法 $^{[7]}$, 气相色谱-三重四极杆质谱法 ${ }^{[8,9]}$ 等. 仪器检测不 仅需要大型仪器设备, 而且还需要经过 “提取一多重净 化一浓缩” 等较为复杂的样品前处理, 检测成本相对较 高，且难以实现现场检测. 在快速检测方面，自 Nebert 等 ${ }^{[10]}$ 提出 POPs 受体致毒机理以来, 基于 2,3,7,8-位取代 的二啞英单体与芳香烃受体(AhR)特异性结合的分析方 法开发一直是 POPs 类化合物速测的研究热点. 欧盟将 细胞生物检测法和试剂盒生物检测法作为 POPs 的篎选

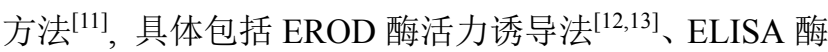
免疫分析法 ${ }^{[14,15]}$ 等. 此外, 基于生物传感原理的方法也 有报道, 如荧光探针 ${ }^{[16]}$ 、荧光定量 $\mathrm{PCR}^{[17]}$ 、电化学生物 传感 ${ }^{[18]}$ 等.

表面增强拉曼光谱(Surface-enhanced Raman Spectroscopy, SERS)是入射光激发纳米尺度贵金属材料产生 的一种拉曼散射增强效应，可实现对单分子的高灵敏 “指纹” 识别，信号增强 $10^{4} \sim 10^{14}$ 倍 ${ }^{[19]}$ ，检测灵敏度可 达 ng 甚至 pg 水平(图 2). 借助 SERS 对目标物的高灵敏 快速识别的技术优势, 实现对 POPs 的分析, 对于丰富 POPs 分析方法具有重要意义和实用价值.

\section{SERS 增强机理}

SERS 增强机理主要包括基于局域表面等离子体共 振(Localized Surface Plasmon Resonance, LSPR)的电磁 (Electro Magnetism, EM)增强和基于电荷转移(Charge Transfer, CT) 的化学增强两种 ${ }^{[20]}$. 一种是电磁场增强, 当目标物接近纳米贵金属表面时，化合物的拉曼散射强 度受贵金属表面 LSPR 的影响而大大增强, 它受纳米粒 子间距、粒径和分布的影响; 另一种是基于 CT 的化学 增强，其理论基础是吸附在粗糙贵金属表面分子的极化 率的改变. 吸附的分子与贵金属表面的原子(族)之间相 互作用，电子在入射光的激发下从分子轨道跃迁到贵金

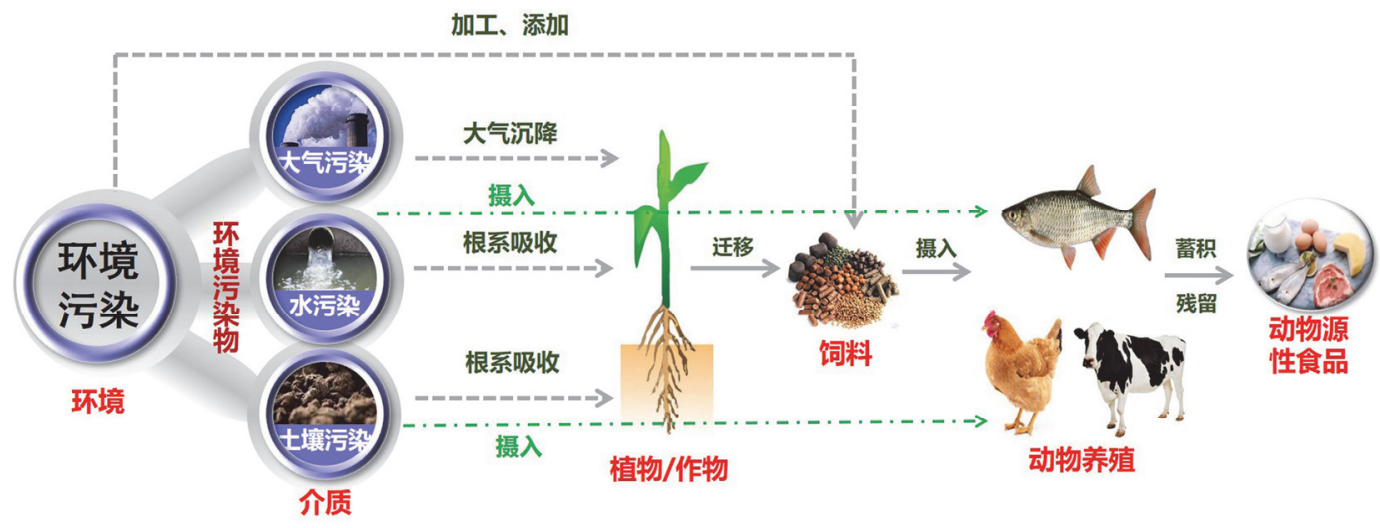

图 1 二噁英类 POPs 化合物迁移途径

Figure 1 Carry-over of dioxins 


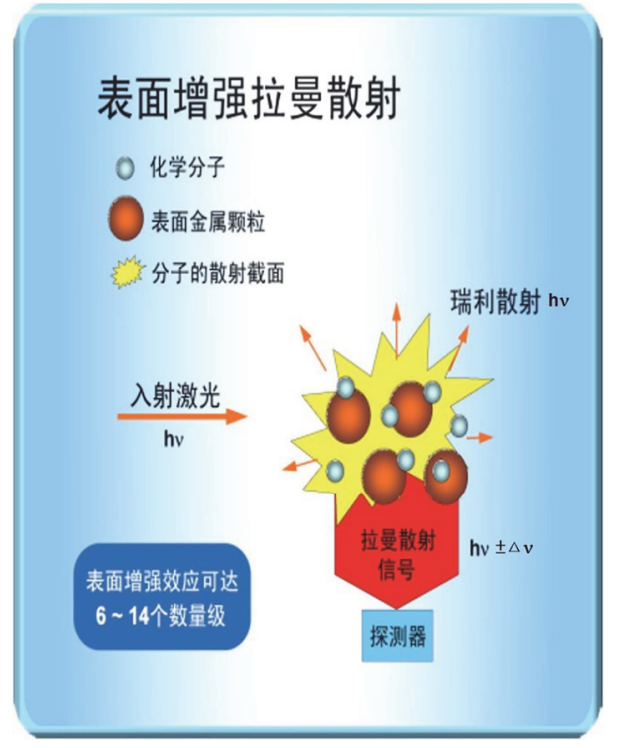

图 2 SERS 增强示意图

Figure 2 Schematic diagram of SERS

属表面的某一能级, 当入射光子能量匹配电子能量和贵 金属电子能差时, 产生共振, 改变了分子极化率, 拉曼 信号得以增强.

迄今为止, 仍没有一个较为完善的理论可以解释所 有的 SERS 现象. 每种增强机制都能部分解释试验结果. 学术界基本认为是两种增强机理模型共同作用的结果, 它们对 SERS 产生的相对贡献率随体系的不同而不同.

\subsection{EM 机理}

大多数的物理类模型认为 SERS 起源于贵金属表面 局域电磁增强, 它们之间的不同在于所提出的局域电场 增强模型不同. 这类模型并不需要金属表面与待测分子 间有化学键作用, 因此无法说明不同吸附分子的 SERS 差异. 表面等离子体共振模型认为, 当粗糙化的贵金属 基体表面受到光照射时, 其表面的等离子体能被激发到 高的能级, 而与光波的电场耦合, 并发生共振, 使金属 表面的电场增强, 产生增强的拉曼散射. 因此实现对 POPs 的高灵敏 SERS 检测, 需要关注如何控制目标 POPs 处于有效的 LSPR 区域. 同时, 还需考虑 EM 增强 受纳米粒子形态和分布的影响, 控制纳米粒子的粒径、 间距及分布也是需要解决的关键问题.

\subsection{CT 机理}

研究者发现许多现象是不能用 EM 机理来解释的. 如当几个单分子层分子连续吸附到贵金属表面时, 首层 吸附分子的 SERS 强度要比其他几层分子的 SERS 强度 强 100 倍以上; 不是所有吸附在贵金属表面的分子都能 产生 SERS 信号, 只有吸附在贵金属表面某些被称为 “热点” (hotspots)的分子才能有强的 SERS 效应. 一些试 验的结果表明, 除了物理增强, 化学增强也在起作用, 化学增强模型提出最多的是电荷转移模型. 它是指分子 吸附在纳米基底表面后, 分子向贵金属表面或者贵金属
表面向分子有电荷转移现象，当激发光波长与电荷转移 态能量相匹配时，电子可从贵金属费米能级附近共振跃 迁到吸附分子上，或者从吸附分子共振跃迁到贵金属 上, 从而改变了分子极化率, 产生 SERS 效应. 然而, POPs 属于疏水性化合物, 难以与基底表面直接发生作 用, 从而给 SERS 分析带来困难. 现有的方法一般通过 基底表面的官能团修饰改性、待分析 POPs 结构改变等 方法, 使得 POPs 与基底发生相互作用, 从而实现 SERS 增强. 但在实际样品基质中 POPs 的 SERS 分析过程中, 面临着如何实现 POPs 在基底表面的吸附，以及如何实 现复杂基质中对目标 POPs 的富集等问题，目前仍未得 到很好的解决.

\section{SERS 分析 POPs 的关键点}

\section{1 分析灵敏度}

SERS 分析的关键点之一是增强基底的构筑, 基底 的形态决定了其 SERS 活性, 也影响对目标 POPs 分析 的灵敏度. (1)在 CT 化学增强和 EM 增强机理共同作用 下，目标物与基底有效 “接触” 或目标物处于有效的 “增 强区域” 是实现对目标 POPs SERS 信号放大的前提. 因 此, 如何 “捕获” 脂溶性较强的 POPs 至基底的 “有效 增强区域” 是需要解决的问题. (2) POPs 在环境、食品 等样品基质中浓度水平低, 光谱信号较弱. 如何构筑高 密度增强 hotspots, 并控制其分布也是 SERS 分析面临 的关键问题，这也为实现 POPs 高灵敏分析提供前提条 件. 随着纳米科学与制造的不断发展, 除了传统的纳米 金/银增强基底外, 各种高密度热点基底的构筑也有报

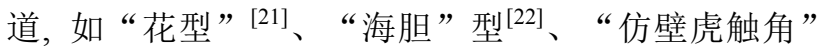

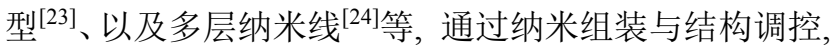
构筑的基底具有高密度增强热点, 提高了对目标物的分 析灵敏度. 但微观形态控制较为复杂, 制备成本也较高.

\section{2 分析稳定性}

纳米金/银等纳米贵金属常常被选择作为 SERS 分 析的增强基底. 这是利用纳米金/银在聚集时产生 hotspots, 在 hotspots 作用下, 实现目标物的 SERS 信号 增强. 但由于聚集过程的随机性, 使得产生的增强热点 分布不规律. 同时，目标物在纳米贵金属表面吸附方式 的随机, 使得增强光谱信号重现性差, 对分析的稳定性 带来很大的影响. 一般来说, 商品化 SERS 基底的稳定 性需大于 1 年时间 ${ }^{[25]}$. 通常通过下列三种方式来提高基 底的稳定性. (1)构筑基于石墨烯(graphene, Gr)的复合纳 米基底. $\mathrm{Gr}$ 是近年来研究较热的一种新型材料, 因其具 有活泼的 $\pi$ 电子，电荷在目标物与 $\mathrm{Gr}$ 间跃迁能量壁垒 较低，与传统贵金属纳米结构一样具有 SERS 属性，被 称为 GERS (Graphene-Enhanced Raman Scattering) 效 应 ${ }^{[26]}$. 深度的氧化作用能使 Gr 表面活性官能团增加, 有利于提高其负载纳米粒子浓度, 增加 hotspots 密度, 从而提高分析灵敏度 ${ }^{[27]}$, 更重要的是“ $\mathrm{Gr}$ 负载贵金属纳 
米” 复合结构在提高 SERS 分析稳定性, 避免使用单一 纳米粒子进行非稳定性增强方面具有很大的应用潜

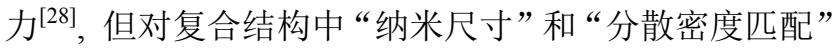
的控制是需要重点解决的问题. (2)构筑固相基底. 利用 先进的纳米制造技术, 如掠入射沉积 (Oblique Angle Depostion, OAD)技术, 在固相基底(玻璃片、硅片等)表 面制备出均匀、高活性纳米银棒阵列 $(A g N R)$. 制得的 $\mathrm{AgNR}$ 均一性好, 批内和批间增强信号强度相对标准偏 差(RSD)分别小于 $10 \%$ 和 $15 \%$, 并用于小分子化合物的 SERS 分析 ${ }^{[29 ~ 31]}$. 但制备成本较高. (3)通过标准化操作 控制 hotspots 分布, 从而提高分析的稳定性. 如通过调 节 $\mathrm{pH}$ 值 ${ }^{[32]}$ 、温度 ${ }^{[33]}$ 等. 此类方法的普适性需要进一步 提高.

\section{3 分析选择性}

环境、食品等实际样品基质, 组成复杂, 众多干扰 物质在基底表面与目标 POPs 产生竞争吸附, 对目标物 SERS 识别干扰严重. 此外, POPs 种类众多, 多为协同 污染, 如何提高分析的选择性非常关键. 通过特异性识 别单元或者快速样品前处理技术与 SERS 联用将大大提

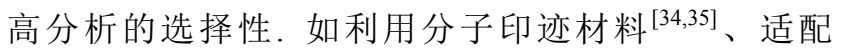
体 $^{[36,37] \text { 、抗体 }}{ }^{[38]}$ 等高特异性识别单元, 或者液液萃 取 ${ }^{[39]}$ 、固相萃取 ${ }^{[40,41]}$ 、薄层色谱 ${ }^{[42]}$ 等分离技术，实现复 杂基质中目标 POPs 的分离与富集.

\section{SERS 分析 POPs 研究进展}

POPs 作为一种主要来源于环境的污染物, 通过迁 移进入食物链. 在环境、食品、农业投入品中含量往往 极低, 再加上基质中其他共存物的干扰, 对 SERS 分析 提出了更高的要求. 一般来说, 对样品中 POPs 的 SERS 分析涉及样品净化处理, 基底对目标物 SERS 增强等两 个关键步骤. 首先, 配套样品前处理手段进行基质净化, 能大大提高目标 POPs 分析的选择性和灵敏度, 但目前, 对样品中 POPs SERS 分析较少报道, 基本都是在实验 室层面对标准品进行研究. 其次, 基底的构筑决定了对 POPs 分析的 SERS 活性, 是 SERS 分析的前提.

\section{1 高密度 “热点” 型基底}

通过设计多维增强热点增加基底的热点密度来实 现目标 POPs 的 SERS 分析. 如 Tang 等 ${ }^{[43]}$ 利用 $\mathrm{Si}$ 为固相 基底, 表面通过电化学沉积制备 $\mathrm{ZnO}$ 纳米雉阵列, 然后 在阵列表面通过离子溅射负载纳米银(AgNPs)粒子构筑 成具有大比表面积的复合 3D 纳米结构(图 3). 该结构产 生三种增强热点, 一是同一 $\mathrm{ZnO}$ 纳米锥阵列上 AgNPs 之间形成的热点; 二是相邻 $\mathrm{ZnO}$ 纳米雉阵列上 AgNPs 彼此之间形成热点; 三是在 $\mathrm{ZnO}$ 纳米雉阵列顶端纳米 银粒子彼此相互作用形成的热点. 在三种不同的增强热 点作用下, 对 PCB-77 的增强因子达 $3.24 \times 10^{7}$, 检测灵 敏度达 $10^{-11} \mathrm{~mol} \cdot \mathrm{L}^{-1}$. 多维增强热点大大提高了分析灵
敏度, 但在对 PCB-77 分析过程中，需要 $10 \mathrm{~h}$ 的吸附时 间，给样品的实际分析带来不便. $\mathrm{Li}$ 等 ${ }^{[44]}$ 在聚丙烯腈纳 米驼峰(PAN-nanohump)表面溅射靶标银, 构筑高密度 热点的 AgNPs@PAN-nanohump 结构, 在 $10^{-5} \mathrm{~mol} \cdot \mathrm{L}^{-1}$ 浓度水平实现 PCB-77 检测, 但灵敏度不高, 这可能与 PCB-77 在基底表面富集行为有关.

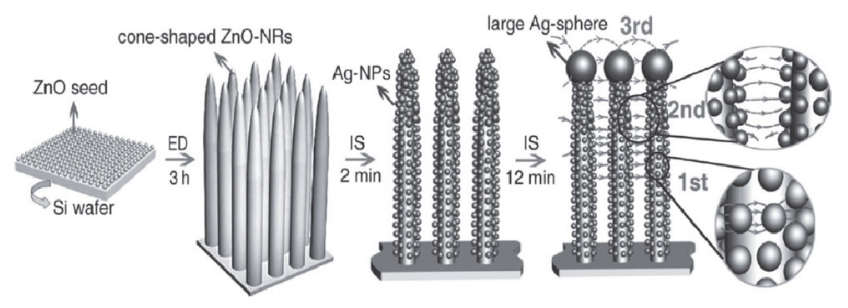

图 $33 \mathrm{D}$ 热点纳米结构组装

Figure 3 Schematic image of the fabrication of 3D hotspots SERS substrate

\section{2 表面修饰型基底}

为有效 “捕获” 目标 POPs 至基底表面以实现拉曼 信号增强, 在基底表面进行功能化修饰(表 2). 通常有以 下几种方式. (1)硫醇类修饰. 修饰在基底表面的长链烷 烃基，通过范德华力实现与 POPs 的相互作用(图 4a $)^{[45]}$. (2)环糊精(-CDs)的官能团化 ${ }^{[46,47]}$. CDs 具有亲脂性内腔 体和亲水性外部结果，能通过静电作用 “捕获” POPs 分子(图 4b). (3)谷胱甘肽(-GSH)修饰, 利用 GSH 中的 -SH 偶联至基底表面 ${ }^{[48]}$. (4)适配体功能化 ${ }^{[36,37]}$. 利用 DNA 或者 RNA 作为识别单元, 实现对目标 POPs 的特 异性识别, 当捕获目标 POPs 后, 偶联在基底表面适配 体的三维构型发现了明显的变化, 其 SERS 位移也随之 发生变化, 通过特征拉曼位移的变化实现目标 POPs 的 特异性识别. 这过程中起关键作用的适配体通常通过指 数富集配体系统净化技术(Systematic Evolution of Ligands by EXponential enrichment, SELEX)进行笁选, 其 工作量大，较为繁琐。 (5)石墨烯负载 ${ }^{[49]}$. 利用石墨烯的 亲脂亲水及富集效应，将贵金属纳米负载于氧化石墨烯 (GO)或还原氧化石墨烯( $\mathrm{rGO}$ )表面，通过 $\pi-\pi$ 共轭作用 富集 POPs, 从而实现 SERS 分析.

\section{3 结构转化}

利用 $-\mathrm{SH}_{3}$ 等官能团的取代或加成反应对目标 POPS 进行化学结构转化, 再进行 SERS 分析. 结构转化后的 衍生物较原型 POPs 分子与增强基底具有更强的作用力, 表现出的特征拉曼吸收强度更高, 便于进行 SERS 识别. 但由于 POPs 类化合物中结构相似的化合物较多, 进行 取代或者加成反应的过程中, 选择性往往较差, 对特定 目标 POPs 的选择性反应不容易控制, 在实际样品基质 中的分析可行性需要进一步提高. Rindzevicius 等 ${ }^{[54}$ 将 PCB-77 通过取代反应转化成 PCB-77-SCH . 研究发现 两者拉曼特征吸收相似，但基底对后者具有更高的 
表 2 基底表面修饰类型

Table 2 Kinds of modification on the surface of SERS substrates

\begin{tabular}{lllcl}
\hline \multicolumn{1}{c}{ 目标物 } & 修饰官能团 & 基质 & 灵敏度 $\left(\mathrm{mol} \cdot \mathrm{L}^{-1}\right)$ & 文献 \\
\hline PCB-47, -77 & alkanethiol & 标液 & $10^{-11}$ & {$[45]$} \\
PCB-40, -54,-65,-80 & $\beta-\mathrm{CD}$ & 土壤 & $-^{a}$ & {$[50]$} \\
PCB-77 & $\beta-\mathrm{CD}$ & 标液 & $10^{-10}$ & {$[51]$} \\
PCB-3, -29, -77 & $\beta-\mathrm{CD}$ & 标液 & $10^{-6}$ & {$[46]$} \\
PCB-77 & HS- $\beta$-CD & 标液 & $10^{-7}$ & {$[47]$} \\
PCB-47 & GSH & 土壤 & - & {$[48]$} \\
PCB-77 & aptamer & 标液 & $10^{-8}$ & {$[36]$} \\
PCB-77 & aptamer & 标液 & $3.3 \times 10^{-8}$ & {$[37]$} \\
PCB-77 & aptamer & 标液 & $10^{-6}$ & {$[52]$} \\
PCB-47, -52, -77 & rGO & 标液 & $10^{-7}$ & {$[49]$} \\
PCB-3, -77 & GO & 标液 & $10^{-4}$ & {$[53]$} \\
\hline
\end{tabular}

${ }^{a}$ 未报道.

SERS 活性，检测灵敏度从 $10^{-5}$ 提高至 $10^{-8} \mathrm{~mol} \cdot \mathrm{L}^{-1}$ (图 5).

\section{5 展望}

利用 SERS 进行 POPs 的分析被证明可行, 但在实 际样品分析还需重点关注以下几个方面.

\section{1 高效样品前处理技术}

实际样品与标准溶液相比, 基质更为复杂. SERS 技 术作为一项识别技术，不具备基质的净化、目标物的分 离功能. 配套高效的样品前处理方法非常重要. 建立的 样品前处理方法需要具备良好的回收率、选择性以及高 效. 目前与 SERS 联用的净化方法较多, 如分子印迹聚 合物 ${ }^{[34,35]}$ 、液液萃取 ${ }^{[39]}$ 、固相萃取 ${ }^{[40,41]}$ 、薄层色谱 ${ }^{[42]}$ 、

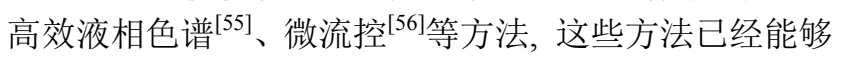
实现与 SERS 联用的要求. 但需要注意的是净化效率与 净化时间之间的关系. 发展配套的快速样品前处理技术 与 SERS 联用, 从实验室层面的标准溶液分析扩展至样 品基质分析, 是 SERS 实用化的必经阶段.

\section{2 基底实用性与检测成本}

SERS 技术已经发展几十年, 在实际检测中还未广 泛应用. 其中关键的技术瓶颈就是基底, 它面临着稳定 性和制备成本等技术难题. 对于溶胶型基底，聚集和沉 淀使得基底活性大大降低，目标物与基底的作用位点和 作用形式随机，产生的增强信号稳定性较差; 固相型基 底的批间和批内增强稳定性也是需要突破的瓶颈难题, 增强 “热点”一般产生在 $2 \mathrm{~nm}$ 的纳米结构之间, 控制固 相纳米尺寸在 $2 \mathrm{~nm}$ 水平较为困难, 此外, 固相基底在暴 露的环境下容易被氧化, 需要保护固相基底表面活性. 如果应用到具体检测分析中，基底的稳定性一般需要至

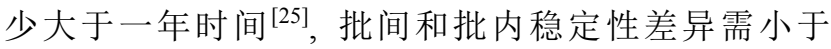
15\%. 此外, POPs 类化合物在基质中的含量水平极低, 对它们的分析属于超痕量分析范畴，如何进一步提高基 底的分析灵敏度和选择性也是下一步研究的重点方向.

在实际应用中, 检测成本也是需要考虑的关键因 素. 其中, 涉及到几个方面. 一是基底制作方面的成本. 为了提高 SERS 分析的稳定性和灵敏度, 利用现代纳米 制造技术是研究热点. 但制造技术依托的大型设备往往 较为昂贵，用于离子溅射的高纯度的银、金等靶材较为 昂贵. 目前，市售的固相溅射基底一般在 $70 \sim 100$ 元/片 $(2 \mathrm{~cm} \times 2 \mathrm{~cm})$, 这对于快速簃查而言, 成本相对较高. 如何降低基底的制作成本也是需要重点考虑的问题. 二 是拉曼光谱仪设备的制造成本. 目前市售的拉曼检测设 备, 国内外都有相似的产品, 设备的价格也参差不齐, 在保证较高光谱分辨率的前提下, 需要降低设备成本, 特别是其中关键零部件的研发, 如 CCD 的研制, 低温冷 却使得仪器信噪比提高, 有利于痕量检测, 激光器的寿 命也是需要考虑的问题. 三是样品分析过程中所用耗材 的成本. 如作为 POPs 识别单位的抗体的制备. 目前国 内没有相关产品, 基本被国外公司垄断, 需要购买进口 抗体.

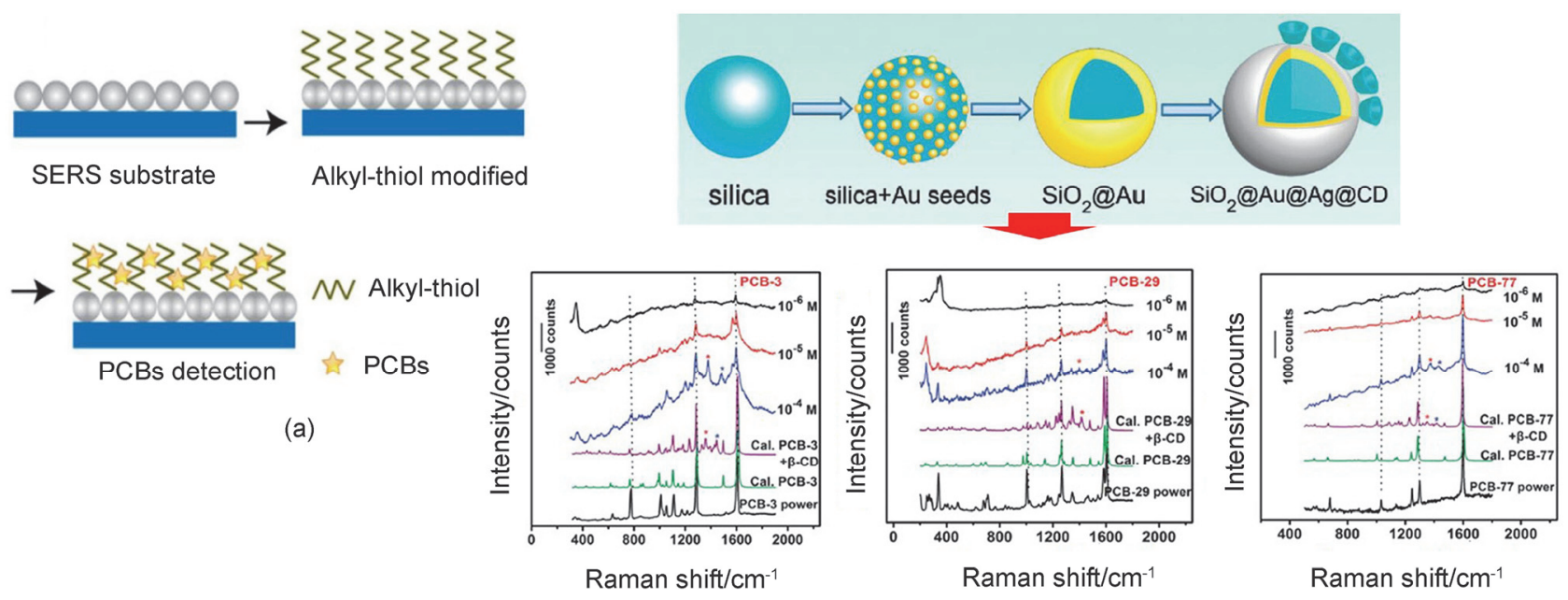

(b)

图 4 (a) 烷硫醇修饰; (b) 环糊精修饰

Figure 4 (a) Alkanethiol modification; (b) $\beta$-CD modification 
(a) PCB-77

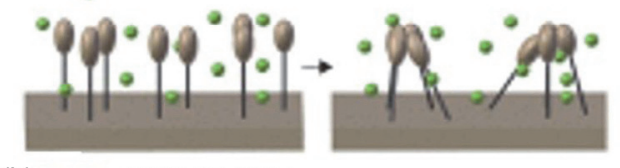

(b) a a PCB-77-SCH
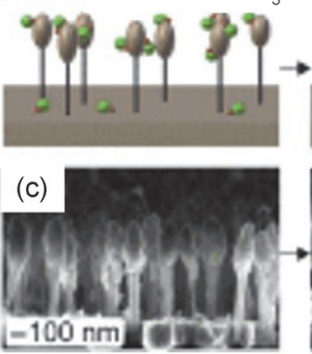
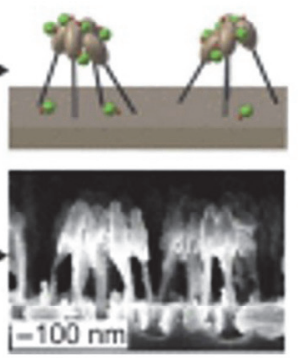

(d)

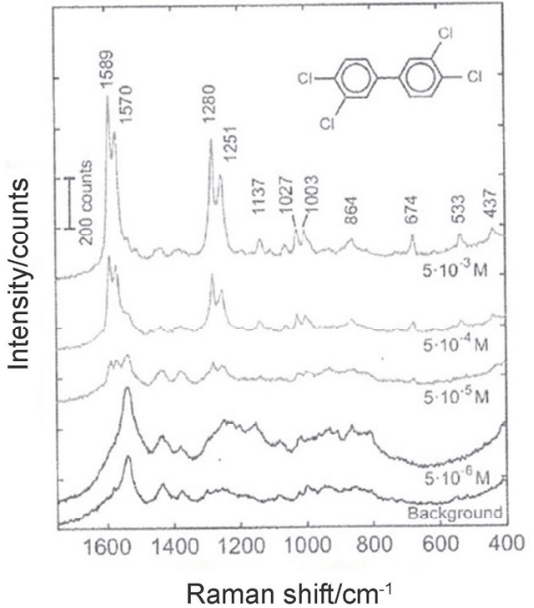

(e)

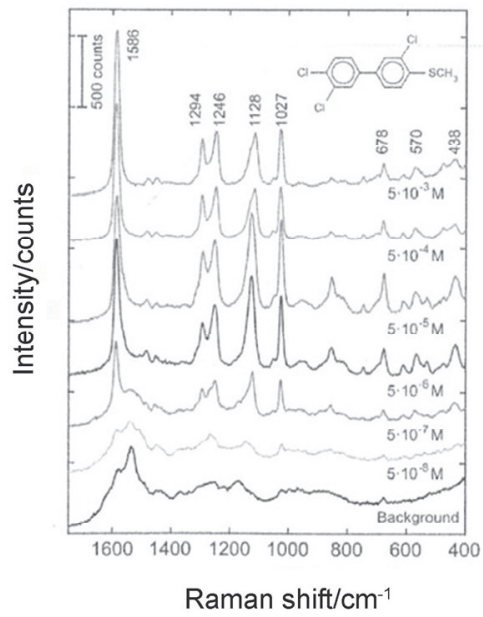

图 5 (a) PCB-77 在基底表面吸附; (b) PCB-77-SCH 在基底表面吸附; (c)基底 SEM 图; (d) $5 \times 10^{-3} \sim 5 \times 10^{-6} \mathrm{~mol} \cdot \mathrm{L}^{-1}$ 浓度范围内 PCB-77 的 SERS 谱图; (e) $5 \times 10^{-3} \sim 5 \times 10^{-8} \mathrm{~mol} \cdot \mathrm{L}^{-1}$ 浓度范围内 PCB-77- $\mathrm{SCH}_{3}$ 的 SERS 谱图

Figure 5 (a) The adsorption of PCB-77 on the base surface; (b) The adsorption of PCB-77-SCH $\mathrm{SH}_{3}$ on the base surface; (c) The SEM images of substrates; (d) SERS spectra of PCB-77 at concentrations of $5 \times 10^{-3} \sim 5 \times 10^{-6} \mathrm{~mol} \cdot \mathrm{L}^{-1}$. (e) SERS spectra of PCB-77-SCH $\mathrm{S}_{3}$ at concentrations of $5 \times 10^{-3} \sim 5 \times 10^{-8}$ $\mathrm{mol} \cdot \mathrm{L}^{-1}$

\section{3 研究对象的扩充与多靶标分析}

目前已有的对 POPs 的 SERS 研究基本集中在 PCBs 类化合物中, PCB-77 的研究报道多于 $95 \%$, 这可能与 PCB-77 高度对称的结构有关, 对其他 POPs 类化合物的 SERS 研究几乎未涉及. 而目前, 国内外的相关法律法 规中, 毒性较大的 17 种 PCDD/Fs 和 12 种 DL-PCBs 都 有各自的 TEF, POPs 的含量水平一般通过毒性当量 (Toxic Equivalent Quantity, TEQ)来表示. 仅仅研究一种 POPs 是远远不够的, 我们可以根据已有的研究基础, 从化合物的结构相似性上考虑去设计合适的增强基底, 分析 POPs 种类和数量进一步进行扩充, 以此满足实际 的应用需求. 此外, 一些新型 POPs 也不断涌现, 如多澳 联苯醚类 $(\mathrm{PBDEs}) 、$ 短链氯化石蜡 $(\mathrm{SCCPs}) 、$ 全氟辛磺酸 (PFOs) 以及阿特拉津 ${ }^{[57]}$ 等, 研究对象函需进一步丰富. 同时, POPs 类化合物种类众多, 多为协同污染, 实现对 多种 POPs 的同时多靶标分析具有重要的意义. 全球环 境监测系统/食品规划部分(GEMS/FOOD)把 7 种指示性 PCBs(PCB 28, 52, 101, 118, 138, 153, 180)的总量作为监 测 PCBs 污染情况的指标; 欧洲食品安全局(EFSA)也将 6 种指示性 PCBs (PCB 28, 52, 101, 138, 153, 180)作为对 食品和饲料进行风险评估的目标物 ${ }^{[58]}$; 我国食品污染 物限量标准(GB 2762-2017)中也明确规定水产动物及其 制品中 7 种指示性 PCBs(PCB 28, 52, 101, 118, 138, 153, 180 )限量为 $0.5 \mathrm{mg} / \mathrm{kg}^{[59]}$. 此外, 我国于 2018 年开始实 施的新版饲料卫生标准(GB 13078-2017)中, 也新增了 饲料及饲料原料中 6 种指示性 PCBs 的限量水平为 10 $175 \mu \mathrm{g} / \mathrm{kg}^{[60]}$. 通过借助化学计量学方法(如主成分分析 PCA 等)可实现对多目标的识别和分析, 提高分析通量.

\section{4 数据处理与软件操作}

对于原始光谱数据的处理方面, 研发具有自主知识 产权的 “算法” 对光谱数据进行处理, 也将大大提高定 性识别、半定量甚至定量分析的准确性. 在实际应用的 过程中，设计简单 “友好”的软件操作界面，将推进 SERS 速测方法在基层的推广应用.

\section{作者简介}

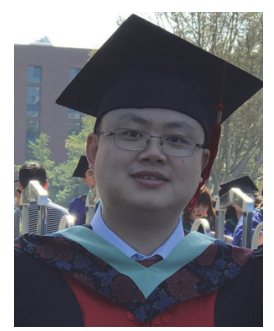

程劼, 博士，副研究员，中国农业科学院农业质量标准与 检测技术研究所, 二噁英污染研究室. 主要从事 SERS 快速分 析技术、POPs 迁移转化规律等研究. 发表 SCI 论文 20 余篇, 授 权发明专利 6 项, 省部级奖励 3 项.

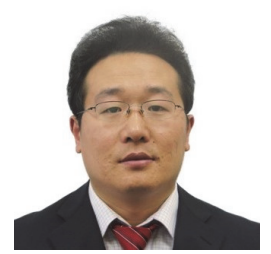

王培龙, 博士, 研究员, 中国农业科学院农业质量标准与 检测技术研究所, 二噁英污染研究室. 主要从事饲料及畜产 品质量安全及 POPs 分析毒理研究. 近年来发表 SCI 论文 30 余篇，授权发明专利 4 项，省部级奖励 5 项. 


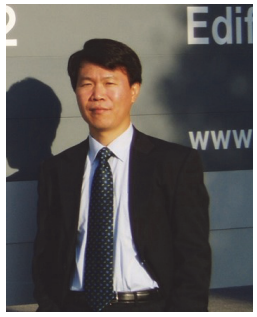

苏晓鸥, 博士, 二级研究员, 中国农业科学院农业质量标 准与检测技术研究所. 主要从事饲料及畜产品质量安全检测 技术研究. 发表 SCI 论文 80 余篇, 授权国家发明专利 10 项, 制定国家和农业行业标准 10 余项, 出版专著 8 部.

\section{References}

[1] Cammilleri, G.; Calvaruso, E.; Pantano, L; Cascio, G. L.; Randisi, B.; Macaluso, A.; Vazzana, M.; Caracappa, G.; Giangrosso, G.; Vella, A.; Ferrantelli, V. Environ. Toxicol. Chem. 2017, 36, 2997.

[2] Adam, T. S. G.; Christoph, A.; Craig, A. B.; Barry, C. P. Mar. Pollut. Bull. 2017, 120, 414

[3] Jamieson, A. J.; Malkocs, T.; Piertney, S.; Fujii, T.; Zhang, Z. L. Nat. Ecol. Evol. 2017, 0051.

[4] Matuszak, M.; Minorczyk, M.; Góralczyk, K.; Hernik, A.; Struciński, P.; Liszewska, M.; Czaja, K.; Korcz, W.; Łyczewska, M.; Ludwicki, J. K. Rocz Panstw Zakl Hig. 2016, 67, 113.

[5] Lv, F.; Gan, N.; Cao, Y.; Zhou, Y.; Zuo, Y.; Dong, Y. J. Chromatogr. A 2017, 1525, 42.

[6] United States Environmental Protection Agency, 2010, Method $1678 \mathrm{C}$.

[7] Xia, D.; Gao, L.; Zheng, M.; Wang, S.; Liu, G. Anal. Chim. Acta 2016, $937,160$.

[8] Dam, G. T.; Pussente, I. C.; Scholl, G.; Eppe, G.; Schaechtele, A.; Leeuwen, S. V. J. Chromatogr. A 2016, 1477, 76.

[9] Li, W.; Liu, D.; Li, J.; Gao, J.; Zhang, C.; Wang, P.; Zhou, Z. Chromatographia 2017, 80, 813.

[10] Nebert, D. W. G.; Goujon, F.; Gielen, J. Nature 1972, 236, 107.

[11] Commission Directive 2002/69/EC. Official J. Eur. Communities 2002a

[12] Levy, W.; Brena, B. M.; Henkelmann, B.; Bernhöft, S.; Pirez, M.; González-Sapienza, G.; Schramm, K. W. Toxicol. in Vitro 2014, 28, 1036.

[13] Crump, D.; Farhat, A.; Chiu, S.; Williams, K. L.; Jones, S. P.; Langlois, V. S. Environ. Sci. Technol. 2016, 50, 3265.

[14] Urbaniak, M.; Tygielska, A.; Krauze, K. Plos One 2016, 11, e0151756.

[15] Ching, L.; Eric, B.; James, L.; Zhang, W. Anal. Bioanal. Chem. 2016, $408,1095$.

[16] Babikian, S.; Li, G. P.; Bachman, M. IEEE Transactions on Components 2017, 7, 846 .

[17] Yang, G.; Zhuang, H.; Chen, H.; Ping, X.; Bu, D. Sens. Actuators B 2015, 214, 152.

[18] Zheng, X.; Li, H.; Xia, F.; Tian, D.; Hua, X.; Qiao, X.; Zhou, C. Electrochim. Acta 2016, 194, 413.

[19] Moskovits, M. J. Chem. Phys. 1982, 77, 4408.

[20] Ruchita, S.; Agrawal, Y. K. Vib. Spectrosc. 2011, 57, 163.

[21] Liang, H. Y.; Li, Z. P.; Wang, W. Z.; Wu, Y. S.; Xu, H. X. Adv. Mater. 2009, 21,4614

[22] Wang, X. S.; Yang, D. P.; Huang, P.; Li, M.; Chen, D.; Cui, D. X. Nanoscale 2012, 24, 7766.

[23] Wang, P.; Wu, L.; Lu, Z. C.; Li, Q.; Yin, W. M.; Ding, F.; Han, H. Y. Anal. Chem. 2017, 89, 2424.

[24] Zhang, B.; Xu, P.; Xie, X. M.; Wei, H.; Li, Z. P.; Mack, N. H.; Han, X. J.; Xu, H. X.; Wang, H. L. J. Mater. Chem. 2011, 21, 2495.

[25] Panneerselvam, R.; Liu, G. K.; Wang, Y. H.; Liu, J. Y.; Ding, S. Y.; Li, J. F.; Wu, D. Y.; Tian, Z. Q. Chem. Commun. 2018, 54, 10.
[26] Xu, W. G.; Mao, N. N.; Zhang, J. Small 2013, 8, 1206.

[27] Cheng, J.; Su, X. O.; Han, C. Q.; Wang, S.; Wang, P. L.; Zhang, S.; Xie, J. C. Sens. Actuators B 2018, 255, 2329.

[28] Cheng, J.; Zhang, S.; Wang, S.; Wang, P. L.; Su, X. O.; Xie, J. C. Food Chem. 2019, 276, 157.

[29] Yao, Y.; Wang, W.; Tian, K. Z.; Ingram, W. M.; Cheng, J.; Qu, L. L.; Li, H. T.; Han, C. Q. Spectrochim. Acta A 2018, 195, 165.

[30] Cheng, J.; Su, X. O.; Yao, Y.; Han, C. Q.; Wang, S.; Zhao, Y. P. Plos one 2016, 11, e0154402.

[31] Han, C. Q.; Chen, J.; Wu, X. M.; Huang, Y. W. Talanta 2014, 128, 293.

[32] Patricia, T. B.; Niklaas, J. B.; Laura, R. L.; Jorge, P. J.; Luis, M. L. M.; Pablo, H. J. Mater. Chem. 2011, $21,16880$.

[33] Bonyár, A.; Csarnovics, I.; Veres, M.; Himics, L.; Csik, A.; Kámán, J.; Balázs, L.; Kökényesi, S. Sens. Actuators B 2018, 255, 433.

[34] Feng, J. Y.; Hu, Y. X.; Grant, E.; Lu, X. Food Chem. 2018, 239, 816.

[35] Zhao, B. W.; Feng, S. L.; Hu, Y. X.; Wang, S.; Lu, X. N. Food Chem. 2019, 276, 366.

[36] Fu, C. C.; Wang, Y.; Chen, G.; Yang, L. Y.; Xu, S. P.; Xu, W. Q. Anal. Chem. 2015, 87, 9555.

[37] Sun, K.; Huang, Q.; Meng, G. W.; Lu, Y. L. ACS Appl. Mater. Interfaces 2016, 8, 5723 .

[38] Lopez, A.; Lovato, F.; Oh, S. H.; Lai, Y. H.; Filbrun, S.; Driskell, E. A.; Driskell, J. D. Talanta 2016, 146, 388.

[39] Zhang, M.; Zhang, X.; Shi, Y.; Liu, Z.; Zhan, J. Talanta 2016, 158 , 322.

[40] Chen, Y.; Zhang, Y.; Pan, F.; Liu, J.; Wang, K.; Zhang, C.; Cheng, S.; Lu, L.; Zhang, W.; Zhang, Z.; Zhi, X.; Zhang, Q.; Alfranca, G.; De la Fuente, J. M.; Chen, D.; Cui, D. ACS Nano 2016, 10, 8169.

[41] Li, B.; Shi, Y.; Cui, J.; Liu, Z.; Zhang, X.; Zhan, J. Anal. Chim. Acta 2016, 923, 66.

[42] Fang, F.; Qi, Y.; Lu, F.; Yang, L. Talanta 2016, 146, 351.

[43] Tang, H. B.; Meng, G. W.; Huang, Q.; Zhang, Z.; Huang, Z. L.; Zhu, C. H. Adv. Funct. Mater. 2012, 22, 218.

[44] Li, Z. B.; Meng, G. W.; Huang, Q.; Hu, X. Y.; He, X.; Tang, H. B.; Wang, Z. W.; Li, F. D. Small 2015, 40, 5452

[45] Bantz, K. C.; Haynes, C. L. Vib. Spectrosc. 2009, 50, 29.

[46] Lu, Y. L.; Yao, G. H.; Sun, K. X.; Huang, Q. Phys. Chem. Chem. Phys. 2015, 17, 21149.

[47] Zhu, C.; Meng, G. W.; Huang, Q.; Li, Z. B.; Huang, Z. L.; Wang, M. L.; Yuan, J. P. J. Mater Chem. 2012, 22, 2271.

[48] Arocikia, J. D.; Parimaladevi, R.; Vasant, S. G.; Umadevi, M. J. Clust. Sci. 2018, 29, 281.

[49] Shanta, P. V.; Cheng, Q. ACS Sensors 2017, 6, 817.

[50] Jency, D. A.; Umadevi, M.; Sathe, G. V. J. Raman Spectrosc. 2015 $46,377$.

[51] Xu, W.; Meng, G. W., Huang, Q.; Hu, X. Y.; Huang, Z. L.; Tang, H. B.; Zhang, J. X. Appl. Surf. Sci. 2013, 271, 125.

[52] Lu, Y. L.; Huang, Q.; Meng, G. W.; Wu, L. J.; Zhang, J. J. Analyst 2014, 139, 3083.

[53] Zhang, C. Y.; Hao, R.; Fu, Y. Z.; Zhang, H.; Moeendarbari, J. S.; Peckering, C. S.; Hao, Y. W.; Liu, Y. Q. Appl. Surf. Sci. 2017, 400, 49.

[54] Rindzevicius, T.; Barten, J.; Vorobiev, M.; Schmidt, M. S.; Castillo, J. J.; Boisen, A. Vib. Spectrosc. 2017, 90, 1.

[55] Jiao, C. L.; Wang, W.; Liu, J.; Yuan, Y. X.; Xu, M. M.; Yao, J. L. Acta Chim. Sinica 2018, 76, 526. (焦岑蕾, 王炜, 刘娇, 袁亚仙, 徐敏敏, 姚建林, 化学学报, 2018, 76, 526.)

[56] Liu, J.; Sun, H. L.; Yin, L.; Yuan, Y. X.; Xu, M. M.; Yao, J. L. Acta Chim. Sinica 2019，77，257. (刘娇，孙海龙，印璐，袁亚仙，徐敏 敏, 姚建林, 化学学报, 2019, 77, 257.)

[57] Jia, F. L.; Liu, J.; Zhang, L. Z. Acta Chim. Sinica 2017, 75, 602. (贾 法龙, 刘娟, 张礼知, 化学学报, 2017, 75, 602.)

[58] Commission Directive 277/2012/EC. Official J. Eur. Communities 2012.

[59] National standards of the People's Republic of China, GB/T 2762-2017. (中华人民共和国国家标准, GB/T 2762-2017.)

[60] National standards of the People's Republic of China, GB/T 13078-2017. (中华人民共和国国家标准, GB/T 13078-2017.) 